\title{
Posttraumatische Belastungsstörungen bei Schizophrenie
}

\author{
Post-Traumatic Stress Disorder in Schizophrenia
}

Bibliografie

DOI 10.1055/s-2006-951941

Psychiat Prax 2007; 34: 55- 57

(c) Georg Thieme Verlag KG

Stuttgart · New York .

ISSN 0303-4259

Korrespondenzadressen

PD Dr. med. Dipl.-Psych.

Roland Vauth

Psychiatrische Poliklinik des

Universitätsspitals Basel

Claragraben 95

4005 Basel, Schweiz

rvauth@uhbs.ch

\section{PD Dr. med. habil. Ronald}

\section{Bottlender}

East London and the City University Mental Health NHS

Trust, Department of

Psychiatry, Newham Centre

for Mental Health

Glen Road, Cherry Tree Way

London E13 8SP

Ronald.Bottlender@elcmht. nhs.uk

\section{Unterschätzter Einfluss posttrauma- tischer Belastungsstörungen auf Schizophrenie}

Die Ausgangslage: Posttraumatischen Belastungsstörungen (PTBS) und schizophrenen Erkrankungen wurden in den vergangenen Jahren v.a. unter drei Blickwinkeln zusammengebracht [1]: a) Einmal kann PTBS als Folge der Erfahrung psychotischer Positivsymptome und von ungünstigen Behandlungserfahrungen auftreten. b) Weiter kann sich eine PTBS als Vulnerabilitätsfaktor für Ausbruch und Rückfall schizophrener Erkrankungen auswirken. c) Schließlich kann sich eine PTBS als Chronifizierungsfaktor schizophrener Symptome auswirken; die mit PTBS assoziierte Meidung der Auseinandersetzung mit erkrankungsbezogenen oder biografischen traumatisierenden Erfahrungen begünstigt einen auf Leugnung der Erkrankung ausgerichteten „Sealing over"-Stil der Krankheitsverarbeitung; hierdurch kann eine PTBS damit indirekt zu unzureichender Compliance und Inanspruchnahme psychiatrischer Hilfsangebote sowie zur Entwicklung sekundärer Suchterkrankungen und Depressionen beitragen.

Epidemiologie: Die Häufigkeit von PTBS bei schizophrenen Erkrankungen ist grundsätzlich gegenüber der Allgemeinbevölkerung erhöht, auch bei strengeren methodischen Kriterien (z. B. standardisiertes Fremd- statt ausschließlich Selbstrating bei der Erfassung der PTBS, homogenere Stichproben ausschließlich schizophrener Patienten). Die Prävalenz liegt zwischen 4-13\% [2] und $28-29 \%$ [3]. Diese höhere Prävalenz ist nicht spezifisch für Schizophrenie, sondern findet sich auch bei bipolaren, depressiven und schizoaffektiven Störungen sowie schweren Persönlichkeitsstörungen wie Borderline-Störungen [4], dabei ist die Prävalenz bei affektiven Erkrankungen am höchsten [4]: So fanden z. B. Mueser et al in der zitierten Arbeit in einer repräsentativeren und grö-
Beren Stichprobe von ca. 780 ambulanten und stationären Patienten in insgesamt fünf Studienzentren eine Punktprävalenz von 35\% über alle Patientengruppen. Die Prävalenz in der Gruppe der schizophrenen Störungen, war mit 29\% geringer als bei den übrigen Störungen: Schizoaffektive Störungen wiesen eine Rate von $37 \%$, bipolare Störungen von 39\% und majore Depression sogar von $45 \%$ auf.

Psychotische Erfahrung und Zwangsbehandlung als PTBS-Ursache: Obwohl die Prävalenz von PTBS bei schizophrenen Störungen insgesamt niedriger ist als bei anderen schweren seelischen Erkrankungen, liegt im Fall komorbider PTBS bei einer schizophrenen Störung im Vergleich zu affektiven Psychosen die Rate derjenigen höher, die psychotisches Erleben oder auch Umstände der Behandlung als traumatisierend angeben (Zwangseinweisung, -medikation, Trennung von Sicherheit stiftenden Angehörigen bei Akutbehandlung). So erlebten $60 \%$ der Patienten mit einer schizophrenen Störung wahnhafte und halluzinatorische Erlebnisse als traumatisierend, während dies nur bei $15 \%$ der bipolaren Patienten der Fall war [5].

PTBS als Vulnerabilitätsfaktor für Ausbruch und Rückfall: Belege für die Bedeutung von PTBS als unspezifischer Vulnerabilitätsfaktor auf der Grundlage der Forschung zum heuristischen Vulnerabilitäts-Stress-Kompetenz-Modell sind vorhanden, wenn auch eher indirekt: Der Zusammenhang von Stress und Symptomexazerbation bei schizophrenen Störungen wurde vielfach nachgewiesen [6,7]. Im Rahmen des Vulnerabilitäts-Stress-Modells psychotischer Rückfälle wurden Traumata und PTBS primär als interne Stressoren gesehen, die Erstauftritt schizophrener Störungen und deren Rückfälle begünstigen [4]: Das Vulnerabilitäts-Stress-Modell sagt voraus, dass ständiges Wiedererleben und Wiedererinnern traumatisierender Erinnerungen (flashbacks, Intrusionen) und die ständige Alarmbe- 
reitschaft des anhaltend erhöhten Erregungsniveaus einer PTBS als interne Stressquellen wirken. Umgebungsfaktoren (z. B. unterstützendes soziales Umfeld, spezifische psychiatrisch-psychotherapeutische Hilfsangebote), Attributions- (z.B. Entwicklung von Schuld oder Scham) und Bewältigungsmuster (Vermeidungsverhalten) wird ein moderierender Einfluss zugewiesen. Diese Variablen beeinflussen, ob das Vorliegen einer PTBS unspezifisch den (Wieder-)Ausbruch einer schizophrenen Störung begünstigt [8]. Biografische Traumata werden als nosologisch unspezifischer Risikofaktor für die Entwicklung einer späteren PTBS oder einer späteren schizophrenen Störung gesehen [9].

PTBS als Chronifizierungsfaktor schizophrener Erkrankungen [1]: Die Evidenz hierfür ist eher indirekt, insofern die Studien meist Querschnitts- und retrospektiven Charakter haben, was nicht - wie im Modell postuliert - kausal gedeutet werden kann. Der Zusammenhang scheint auch komplexer zu sein, denn die Befundlage ist z.T. inkonsistent: Neben einer höheren Ausprägung von Positivsymptomatik bei gleichzeitiger PTBS fand sich auch gleich hoch ausgeprägte Positivsymptomatik. Weiter kann PTBS-bedingte soziale Meidung für einen ungünstigen Erkrankungsverlauf relevant sein. Denn sozialer Rückzug ist ein Risikofaktor für stärkere Rückfall- und Rehospitalisierungsgefährdung, da er mit Mangel an sozialer Unterstützung, an Überprüfung eigener Einschätzung und an Stimulation einhergeht. Auch diese Evidenz ist indirekt. Prospektive Längsschnittstudien zum Zusammenhang mit PTBS-bedingter Meidung und vermehrtem Rückfall fehlen. Schließlich gab es vor Jahren Ansätze, die Negativsymptomatik auch als Reaktion auf die traumatisierende Erfahrung der floriden Psychose sahen, während in neuerer Zeit Negativsymptomatik eher neurobiologisch erklärt wird. Als weiteres indirektes Argument wird in der Literatur angeführt, dass bei schizophrenen Störungen mit komorbider PTBS auch eine erhöhte Vulnerabilität für die Entwicklung von Suchterkrankungen besteht. Diese Patienten wiesen nämlich häufigere und schwerere Traumatisierungen in der Kindheit auf. Zurückgeführt wird dies auf den gemeinsamen ätiologischen Faktor von Schizophrenie und Sucht sowie die CRH-induzierte Stress-Sensitivierung einer überschießend antwortenden Hypophysen-Hypothalamus-Nebennierenrindenachse der endokrinen Stressregulation. Trotz einer gewissen Plausibilität des Zusammenhangs fehlen aber auch hier Längsschnittstudien, die die Kausalität erhärten könnten.

Zusammenfassend spricht also die Studienlage dafür, PTBS bei schizophrenen Erkrankungen in den diagnostischen Abklärungsprozess zu integrieren, um zu überprüfen, ob dies als Fokus gezielter kognitiv-verhaltenstherapeutischer Interventionen [1] und damit zur Verbesserung der Rezidivprophylaxe genutzt werden kann. Für diesen pragmatischen Ansatz ist unerheblich, dass es sich um einen nosologisch eher unspezifischen Vulnerabilitätsfaktor handelt. Ebenso unerheblich ist, dass psychotisches Erleben und Behandlungsmaßnahmen zwar bei Menschen mit Schizophrenie häufiger zu PTBS führt als bei anderen psychotischen Störungen wie affektiven Erkrankungen, insgesamt aber wohl andere Traumatisierungen sehr viel häufiger der komorbiden PTBS bei schizophrenen Erkrankungen zugrunde liegen.

\section{Posttraumatische Belastungsstörungen: Keine typische Folge von Schizophrenie $\nabla$}

Zweifelsohne ist eine Psychose für die meisten Betroffenen eine leidvolle Erfahrung. Und manche Betroffene mögen ihre Psychose sogar als so traumatisierend empfinden, dass sie postpsychotisch Symptome einer posttraumatischen Belastungsstörung (PTSD) entwickeln. Dieses zu bestreiten könnte nur unklug sein. Dass aber mehr als 50\% der schizophrenen Patienten nach ihrer Psychose eine PTSD aufweisen sollen, wie dies einige Studien zu dieser Thematik berichten $[10,11]$, widerspricht der klinischen Erfahrung wohl der meisten Psychiater und muss insofern kritisch hinterfragt werden.

Es geht im Folgenden also nicht darum, prinzipiell zu bezweifeln, dass postpsychotisch eine PTSD auftreten kann. Infrage gestellt werden muss aber, dass dies häufig oder gar bei den meisten schizophrenen Patienten der Fall sei. Befunde, die letzteres suggerieren, sind, wie nachfolgend dargelegt werden soll, am ehesten als methodisch bedingte Artefakte im Kontext einer Überstrapazierung des Komorbiditätsprinzips und zeitgeistabhängiger diagnostischer Moden zu verstehen.

Zunächst einmal ergibt sich bei der hier zur Debatte stehenden Thematik die Frage, ob eine Psychose überhaupt ein Trauma im Sinne des PTSD Konzeptes sein kann oder nicht. Die Erörterung dieser prinzipiellen Frage ist vielschichtig und kann hier aus Platzgründen nur kurz angerissen werden. Hauptargument der Befürworter dieser Frage ist, dass die Erlebnisse i.R. einer Psychose für den Betroffenen quasi Realitätscharakter besitzen und insofern kein Unterschied besteht, ob das traumatische Ereignis sich faktisch ereignete oder „nur“ psychotisch erlebt wurde [8]. Hier möchte man einwenden, dass zwischen beiden Arten traumatischer Ereignisse (real vs. psychotisch) wie auch zwischen den subjektiven Erlebens- und Verarbeitungsmodi beider Traumata so erhebliche qualitative Unterschiede bestehen, dass es fraglich erscheint, ob diesbezüglich eine Gleichsetzung ohne weiteres möglich ist. Nimmt man dies trotzdem einmal positiv an, wäre natürlich prinzipiell weiter zu fragen, warum nicht auch andere schwere psychische Erkrankungen oder z.B. auch Albträume zu PTSD führen können.

Abgesehen von dieser prinzipiellen Problematik ergeben sich bei der Diagnostik der PTSD im Kontext einer Schizophrenie aber auch zahlreiche andere, eher methodisch bedingte Probleme, die von den meisten Studien zu dieser Thematik nur unzureichend berücksichtigt wurden.

Ein sehr offensichtliches methodisches Problem findet sich beispielsweise bezüglich der Überlappung der i.R. einer Schizophrenie typischerweise vorkommenden psychotischen, negativen und depressiven Symptome mit jener Symptomatik, die im Kontext einer PTSD zu erwarten ist [12]. Werden diese Symptombereiche losgelöst vom Kontext einer Schizophrenie und der diesbezüglich zu erwartenden Symptomatik betrachtet, sind die Symptomkriterien einer PTSD unter der Annahme, dass eine Psychose ein Trauma in der Konzeptualisierung der PTSD darstellt, mitunter schnell erfüllt. So können sich aufdrängende, belastende Gedanken an die Psychose im Sinne intrusiver Gedanken interpretiert werden. Ebenso sind Erinnerungslücken oder bestimmte Übererregungssymptome und insbesondere Vermeidungsverhalten oder emotionale „Taubheit“ im Sinne einer Affektverflachung Symptome, die bei einer Schizophrenie häufig beobachtet werden können. Ob man diese Symptome nun als postpsychotische PTSD, postpsychotische Depression oder Residualsymptomatik beurteilt, hängt wesentlich von der 
konzeptuellen Perspektive ab, aus der heraus man diese Symptome betrachtet und schlussendlich interpretiert. Eine spezifische und reliable Zuordnung der Symptomatik zu ausschließlich einer der genannten Diagnosen ist nicht möglich. Nichtsdestotrotz wird in Studien zu PTSD bei Schizophrenie oft so getan, als ob man dies könne. Da das schizophrene Erscheinungsbild sehr vielgestaltig ist, könnte man natürlich neben einer PTSD zudem noch zahlreiche andere komorbide Störungen diagnostizieren. Und tatsächlich wird heute ja auch oft schon so verfahren. Dies geschieht in Übereinstimmung mit dem sogenannten Komorbiditätsprinzip und der momentan in der Psychiatrie favorisierten rein deskriptiven, atheoretischen und im Prinzip anosologischen Diagnostik. Diese Prinzipien erscheinen angesichts der großenteils immer noch ungenügenden empirischen Fundierung der nosologischen Konzepte psychiatrischer Krankheitsbilder zwar durchaus als sinnvoll, sind aber eben auch unverkennbarer Ausdruck der Schwäche dieser Konzepte. Ein Umstand, der eigentlich eher zu bescheidener diagnostischer Zurückhaltung, denn zu ausufernder Polydiagnostik komorbider Erkrankungen und spekulativen Interpretationen gemahnen sollte.

Ein weiteres methodisches Problem bei der Behandlung des Themas Trauma und Psychose ist in der Abgrenzung zwischen traumatisierenden Effekten der Psychose selbst, und jenen von Ereignissen während der Psychose zu sehen. Zwangsbehandlung, Fixierung oder Isolierung i. R. der Behandlung einer Schizophrenie werden von den Betroffenen in der Tat vielfach als traumatisierend erlebt und können insofern natürlich prinzipiell auch eine PTSD nach sich ziehen $[8,12]$. Ursache der PTSD wären dann aber Zwangsmaßnahmen während der Behandlung und nicht die Psychose an sich. Diese Differenzierung ist in vielen Studien zu dieser Thematik nicht erfolgt. Auch die Tatsache, dass traumatische Ereignisse Psychosen hervorrufen können, was zu einer symptomatischen Überlappung der Folgen des traumatischen Ereignisses mit jenen der ausgelösten Psychose führt oder die insgesamt hohe Prävalenz von lange vor der Psychose erlebten Traumatisierungen, die i.R. der Psychose bisweilen reaktualisiert werden können, fanden in Studien zu PTSD infolge Schizophrenie nur unzureichende oder gar keine Beachtung.

Wie anhand des zuvor Dargestellten deutlich wird, können Symptome von Traumatisierungen im Kontext der Schizophrenie vielfältige Ursachen haben und methodisch schwer von Symptomen/Syndromen des schizophrenen Krankheitsbildes selbst abgrenzbar sein. Traumata vor, während und nach einer
Psychose stellen durchaus häufige Ereignisse dar, die bei der Diagnostik und Therapie schizophrener Patienten, tendenziell zu wenig berücksichtigt werden $[8,13]$. Posttraumatische Belastungsstörungen infolge des psychotischen Erlebens i.R. einer Schizophrenie sind diesbezüglich allerdings nur ein Teilaspekt, der in seiner Häufigkeit gegenwärtig wahrscheinlich erheblich überschätzt wird. Nichtsdestotrotz verdient das Thema Trauma und Psychose an und für sich aber sicherlich berechtigterweise mehr Beachtung als dies in der Vergangenheit der Fall war.

\section{Literatur}

1 Vauth R, Nyberg E. Posttraumatische Belastungsstörungen bei schizophrenen Störungen: Rarität oder Risiko für Compliance und Erkrankungsverlauf? Fortschr Neurol Psychiatr, 2006; in press

2 Braga RJ, Mendlowicz MV, Marrocos RP, Figueira IL. Anxiety disorders in outpatients with schizophrenia: prevalence and impact on the subjective quality of life. J Psychiatr Res 2005; 39 (4): 409-414

3 Harrison CL, Fowler D. Negative symptoms, trauma, and autobiographical memory: an investigation of individuals recovering from psychosis. J Nerv Ment Dis 2004; 192 (11): 745 - 753

4 Mueser KT, Salyers MP, Rosenberg SD, Goodman LA, Essock SM, Osher FC, Swartz MS, Butterfield MI. Interpersonal trauma and posttraumatic stress disorder in patients with severe mental illness: demographic, clinical, and health correlates. Schizophr Bull 2004; 30 (1): 45 - 57

5 Kennedy BL, Dhaliwal N, Pedley L, Sahner C, Greenberg R, Manshadi MS. Post-Traumatic Stress Disorder in subjects with schizophrenia and bipolar disorder. J Ky Med Assoc 2002; 100 (9): 395 - 399

6 Norman RMG, Malla A. Stressful life events and schizophrenia: I A review of the research. Br J Psychiatry 1993; 162: 161-166

7 Bebbington P, Kuipers $L$. The clinical utility of expressed emotion in schizophrenia. [Review] [74 refs]. Acta Psychiatr Scand Suppl 1994; 382: $46-53$

8 Morrison AP, Frame L, Larkin W. Relationships between trauma and psychosis: a review and integration. Br J Clin Psychol 2003; 42 (Pt 4): $331-353$

9 Janssen I, Krabbendam L, Bak M, Hanssen M, Vollebergh W, Graaf R de, Os J van. Childhood abuse as a risk factor for psychotic experiences. Acta Psychiatr Scand 2004; 109 (1): $38-45$

10 Frame L, Morrison AP. Causes of posttraumatic stress disorder in psychotic patients. Arch Gen Psychiatry 2001; 58 (3): 305-306

11 Shaw K, McFarlane AC, Bookless C, Air T. The aetiology of postpsychotic posttraumatic stress disorder following a psychotic episode. J Trauma Stress 2002; 15 (1): 39-47

12 Priebe S, Broeker M, Gunkel S. Involuntary admission and posttraumatic stress disorder symptoms in schizophrenia patients. Compr Psychiatry 1998; 39 (4): 220-224

13 Seedat S, Stein MB, Oosthuizen PP, Emsley RA, Stein DJ. Linking posttraumatic stress disorder and psychosis: a look at epidemiology, phenomenology, and treatment. J Nerv Ment Dis 2003; 191 (10): 675681 